\title{
Survival differences between chemotherapy and chemoradiotherapy in metastatic esophageal cancer: a propensity score-matched study based on the SEER database
}

\author{
Yue Shao $^{1 \#}$, Min Zhang ${ }^{1 \#}$, Liu Ye ${ }^{2}$, Dan Chen ${ }^{1}$, Qing-Chen Wu ${ }^{1}$, Cheng Zhang ${ }^{1}$ \\ ${ }^{1}$ Department of Cardiothoracic Surgery, The First Affiliated Hospital of Chongqing Medical University, Chongqing, China; ${ }^{2}$ The First Branch, The \\ First Affiliated Hospital of Chongqing Medical University, Chongqing, China \\ Contributions: (I) Conception and design: C Zhang; (II) Administrative support: QC Wu; (III) Provision of study materials or patients: None; (IV) \\ Collection and assembly of data: Y Shao, L Ye; (V) Data analysis and interpretation: Y Shao, M Zhang; (VI) Manuscript writing: All authors; (VII) \\ Final approval of manuscript: All authors. \\ \#These authors contributed equally to this work. \\ Correspondence to: Cheng Zhang, MD. The First Affiliated Hospital of Chongqing Medical University, No. 1 Youyi Road, Yuzhong District, \\ Chongqing, China. Email: zhangchengcqmu@126.com.
}

Background: The aim of this study was to explore the impact of chemotherapy (CT) and
chemoradiotherapy (CRT) on prognosis in metastatic esophageal cancer (mEC) patients.
Methods: Information of patients with mEC from 2010 to 2016 was retrieved from the Surveillance,
Epidemiology, and End Results (SEER) database. Demographic and clinical data between CT and CRT
groups were compared. Propensity score matching (PSM) analysis was used to reduce the influence of
potential confounding factors. Multivariate Cox regression analysis was used to evaluate prognostic factors.
Moreover, interaction tests and survival analyses were performed to determine whether pathological type
conferred any survival benefits in subgroups. Results: A total of 3,352 mEC patients were recruited including 1,697 CT patients and 1,655 CRT patients. In multivariable Cox regression, marital status, gender and pathological type were identified as independent prognostic factors for mEC. There was no statistical significance between the CT group and CRT group in overall survival (OS) and cancer-specific survival (CSS) in the matched and unmatched cohort. In subgroup analyses, patients with esophageal adenocarcinoma (EAC) undergoing CT had favorable prognosis. However, in the subgroup of esophageal squamous cell carcinoma (ESCC), patients in the CT group had worse outcomes compared with patients in the CRT group.

Conclusions: Patients with EAC and ESCC could respectively benefit from CT and CRT. Besides, we recommend individualizing the treatment strategy for $\mathrm{mEC}$ based on the pathological type.

Keywords: Prognosis; metastatic esophageal cancer (mEC); chemoradiotherapy; chemotherapy

Submitted Oct 27, 2020. Accepted for publication Jan 18, 2021.

doi: 10.21037/apm-20-2126

View this article at: http://dx.doi.org/10.21037/apm-20-2126

\section{Introduction}

Esophageal cancer (EC) is the ninth most common type of cancer (1), and is comprised of two main pathological types namely, esophageal adenocarcinoma (EAC) and esophageal squamous cell carcinoma (ESCC). EC has a complex lymphogenous hematological pathway that leads to distant metastasis and cancer-related death (2). At the time of initial diagnosis, cancer in $35-50 \%$ of patients had metastasized to distant lymph nodes or organs $(1,3,4)$. Tanaka $e t a l$. reported that the 5 -year relative survival of metastatic esophageal cancer ( $\mathrm{mEC}$ ) was less than $5 \%$ and only $11.2 \%$ of patients could survive for more than 


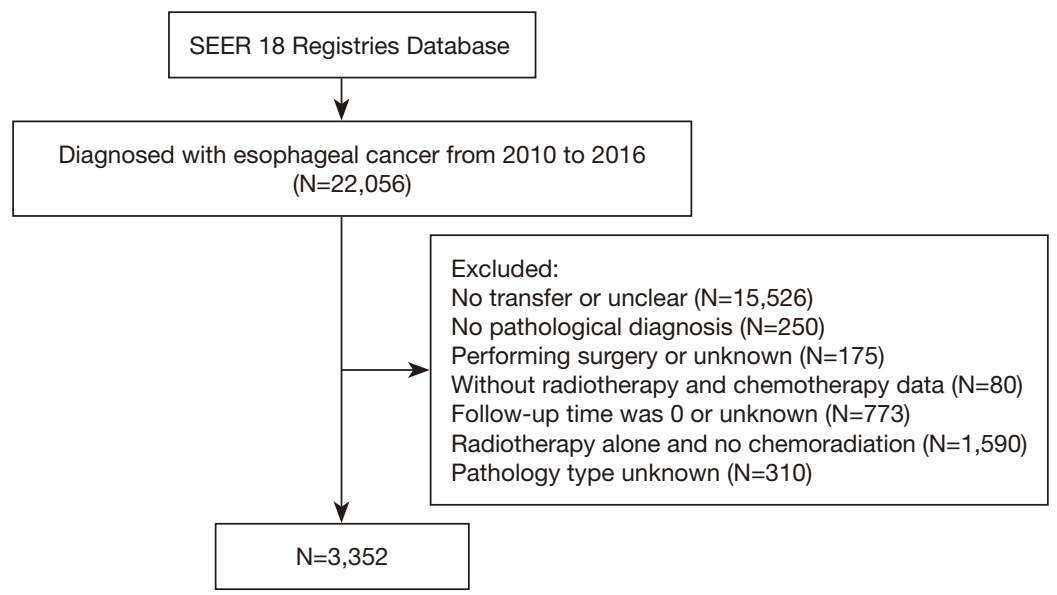

Figure 1 The flow diagram of the selection process for the study.

2 years (5).

Chemotherapy (CT) and chemoradiotherapy (CRT) play major roles in palliative therapy and are currently the primary modes of treatment for mEC $(6,7)$. However, it is not clear whether there is a survival difference between $\mathrm{CT}$ and CRT in the treatment of mEC. Further research is needed to explore better treatment strategies for mEC. The aim of this study was to compare the OS and CSS of mEC patients who underwent CT or CRT and select the subgroups which benefit more from CT or CRT based on the Surveillance, Epidemiology, and End Results (SEER) database. We present the following article in accordance with the STROBE reporting checklist (available at http:// dx.doi.org/10.21037/apm-20-2126).

\section{Methods}

All patients in the current study were obtained from the SEER database (http://seer.cancer.gov/). The SEER cancer data represent a large portion of the American population (30\%), covering 18 population-based registries. Relevant information of patients was retrieved through the SEER*Stat software (Version 8.3.6). The study was conducted in accordance with the Declaration of Helsinki (as revised in 2013). All data were extracted from the public database and did not involve personally identifiable information, so informed consent was not required.

Relevant information was extracted from populationbased database. These include identification, age, race, marital status, pathological type, grade, gender, tumor location, radiotherapy, CT and survival month.

\section{Data collection}

Primary EC was selected using the International Classification of Diseases for Oncology (ICD-O-3) codes 150 to 155 and 158 to 159 . Adenocarcinoma and squamous cell carcinoma were respectively identified as $8140-8389$ and $8050-8089$. The upper esophagus codes were defined as the combination of C15.0 (cervical esophagus) and C15.3 (the upper third of the esophagus); the middle esophagus code was C15.4; the lower esophagus codes were C15.2 (abdominal esophagus) and C15.5 (lower esophagus). The marital status of every patient was redefined as either partnered (domestic partner or married) or un-partnered (divorced, separated, single, or widowed). The OS referred to the interval from initial diagnosis to death, regardless of the cause. CSS was defined as the period from diagnosis to death associated with EC.

The inclusion and exclusion criteria of this study are summarized in Figure 1. The inclusion criteria included: primary EC patients aged over 18 years and diagnosed with EC between January 2010 and December 2016. The exclusion criteria included: (I) cases with unknown or negative metastatic status; (II) cases without pathological diagnosis; (III) cases that received surgery or were unclear; (IV) cases without radiotherapy and CT data; (V) cases whose survival time was either missing or 0; (VI) cases who received radiotherapy alone or did not undergo any other treatment; (VII) cases with other pathological types, other than ESCC and EAC.

\section{Statistical analysis}

To minimize possible selection bias, a 1:1 propensity score matching (PSM) was implemented between patients with 
CT or CRT using the nearest neighbor matching algorithm and a caliper of 0.05 based on the R package MatchIt (Figure S1). Independent variables were selected into the propensity model, including age, gender, pathological type, marital status, race, grade and tumor location. The chisquare test was used to evaluate all variables between two groups, represented as numbers and percentages.

We performed multivariable Cox proportional hazards regression analysis to account for potential confounders and screen prognosis-related factors, which were expressed as the adjusted hazard ratio (HR) and $95 \%$ confidence interval (CI). Independent prognostic factors were included in subgroup analysis. Moreover, interaction tests were performed to explore whether any survival benefit conferred by treatment varied across subgroups. The survival estimates were obtained using the Kaplan-Meier method and compared using the log-rank test. Moreover, interaction tests and survival analyses were used to examine the survival benefits of different subgroups in different treatment modes. Statistical significance threshold was set at 0.05 . Statistical analyses were performed using the $\mathrm{R}$ software (version 3.3.4; http://www.r-project.org/).

\section{Results}

\section{Patient}

In total, we enrolled 22,056 EC patients from the SEER database and excluded 18,704 patients based on exclusion criteria. Eventually, we included 3,352 mEC patients as shown in Figure 1, of which 1,697 (50.63\%) and 1, 655 (49.37\%) patients underwent CT and CRT, respectively.

All characteristics between the CT and CRT groups were matched by PSM at a 1:1 ratio. The histograms of propensity scores for patients stratified by therapeutic strategies are shown in Figure S1. Finally, 1,431 patients were enrolled in both groups. Before matching, there were significant differences in race, pathological type and tumor location between the two groups. Patients who underwent CRT were mainly non-whites and had a higher proportion of ESCC, but were less likely to have a lower tumor location (all $\mathrm{P}<0.05$, Table 1). After matching, no significant difference was revealed in residual factors between both groups $(\mathrm{P}>0.05)$, except for tumor location $(\mathrm{P}<0.001)$.

\section{Survival analysis}

Among the 3,352 mEC patients, 2,418 died of all causes.
The 1- and 2-year OS rates were $40.66 \%$ and $18.35 \%$ in the CT group, respectively, compared with $37.24 \%$ and $16.13 \%$ in CRT group (log-rank $\mathrm{P}=0.28$; Figure $2 A$ ). A total of 2, 379 patients with $\mathrm{mEC}$ died of EC. The 1- and 2-year CSS rates were $41.28 \%$ and $19.01 \%$ in the CT group, respectively, compared to $37.66 \%$ and $16.60 \%$ in CRT group (log-rank $\mathrm{P}=0.25$; Figure $2 B$ ). After PSM, there was still no significant difference between both groups in OS and CSS (all $\mathrm{P}>0.05$; Figure 2C,D).

The results of multivariate Cox regression analyses for cohorts before and after matched are shown in Table 2 . Multivariable analyses indicated that un-partnered, male and ESCC were significantly correlated with worse OS and CSS before and after matching (all $\mathrm{P}<0.05$, Table 2). However, in the original model, CRT group displayed no significant differences in OS (HR $=1.02 ; 95 \%$ CI, 0.94-1.11; $\mathrm{P}=0.584)$ and CSS (HR $=1.03 ; 95 \% \mathrm{CI}, 0.94-1.11 ; \mathrm{P}=0.542)$ compared with the CT group. Similar results were observed in the PSM Cox regression model. Other factors were not associated with the prognosis of mEC patients (all $\mathrm{P}>0.05$ ).

When examining the benefit of treatment modes stratified by pathological type, gender, and marital status, other variables are also included in the multivariate Cox regression model (Figure 3). Interaction tests showed that OS and CSS of CT group and CRT group significantly varied across different pathological types $\left(\mathrm{P}_{\text {interaction for }} \mathrm{OS}\right.$ and $\mathrm{P}_{\text {interaction for Css }}<0.001$, Figure 3). CRT-receiving EAC patients were consistently linked with poor $\mathrm{OS}(\mathrm{HR}=1.11$; 95\% CI, 1.01-1.22; $\mathrm{P}=0.031)$ and $\mathrm{CSS}$ (HR $=1.11 ; 95 \% \mathrm{CI}$, 1.01-1.22; $\mathrm{P}=0.026)$ compared with CT-receiving patients. Patients with ESCC undergoing CRT had significantly better OS (HR $=0.80 ; 95 \% \mathrm{CI}, 0.67-0.95 ; \mathrm{P}=0.012)$ and CSS $(\mathrm{HR}=0.80 ; 95 \%$ CI, 0.67-0.95; $\mathrm{P}=0.012)$ than those undergoing $\mathrm{CT}$. Other variables were not related to the prognosis of $\mathrm{mEC}$ patients receiving different treatments.

Kaplan-Meier curves were performed based on EAC and ESCC before and after PSM in order to further confirmed the survival differences between the CRT and CT groups in different pathological types of mEC. The OS and CSS for the EAC patients receiving CT were significantly better than patients receiving CRT before and after PSM (all $\mathrm{P}<0.05$ ), while OS and CSS for the ESCC patients undergoing CRT were significantly better than patients undergoing CT before and after PSM in the Figures 4,5 (all $\mathrm{P}<0.05$ ).

\section{Discussion}

This study demonstrated that ESCC patients who 
Table 1 Comparison of baseline variables between chemotherapy and chemoradiotherapy groups in the original and matched datasets in cases of metastatic esophageal cancer

\begin{tabular}{|c|c|c|c|c|c|c|}
\hline Variables & \multicolumn{3}{|c|}{ Original data set } & \multicolumn{3}{|c|}{ Matched data set } \\
\hline Total & 1,697 & 1,655 & & 1,431 & 1,431 & \\
\hline Marital status & & & 0.127 & & & 0.355 \\
\hline Partnered & $1,031(60.8)$ & $964(58.2)$ & & 854 (59.7) & 863 (60.3) & \\
\hline Unknown & $76(4.5)$ & $63(3.8)$ & & $64(4.5)$ & $49(3.4)$ & \\
\hline Race & & & $<0.001$ & & & 0.981 \\
\hline White & 1,498 (88.3) & 1,362 (82.3) & & $1,253(87.6)$ & $1,247(87.1)$ & \\
\hline Black & $125(7.4)$ & $170(10.3)$ & & $113(7.9)$ & $117(8.2)$ & \\
\hline Gender & & & 0.054 & & & 0.757 \\
\hline Female & $222(14.1)$ & $252(16.7)$ & & 218 (15.2) & $225(15.7)$ & \\
\hline Male & 1,350 (85.9) & 1,258 (83.3) & & $1,213(84.8)$ & $1,206(84.3)$ & \\
\hline Age & & & 0.715 & & & 0.461 \\
\hline$<65$ years & $400(23.6)$ & $400(24.2)$ & & $343(24.0)$ & $361(25.2)$ & \\
\hline$\geq 65$ years & 1,297 (76.4) & 1,255 (75.8) & & 1,088 (76.0) & $1,070(74.8)$ & \\
\hline Grade & & & 0.175 & & & 0.420 \\
\hline G1 & $41(2.4)$ & $47(2.8)$ & & $40(2.8)$ & $35(2.4)$ & \\
\hline ESCC & $286(16.9)$ & $497(30.0)$ & & $286(20.0)$ & $286(20.0)$ & \\
\hline Tumor location & & & $<0.001$ & & & $<0.001$ \\
\hline Lower & $1,245(73.4)$ & $1,045(63.1)$ & & $1,035(72.3)$ & $1,011(70.6)$ & \\
\hline Middle & $139(8.2)$ & $231(14.0)$ & & $131(9.2)$ & $131(9.2)$ & \\
\hline Upper & $24(1.4)$ & $92(5.6)$ & & $21(1.5)$ & $60(4.2)$ & \\
\hline Unknown & $289(17.0)$ & 287 (17.3) & & $244(17.1)$ & $229(16.0)$ & \\
\hline
\end{tabular}

*, Asian, Pacific Islander and American Indian/Alaska Native. CT, chemotherapy; CRT, chemoradiotherapy; ESCC, esophageal squamous cell carcinoma; EAC, esophageal adenocarcinoma. 

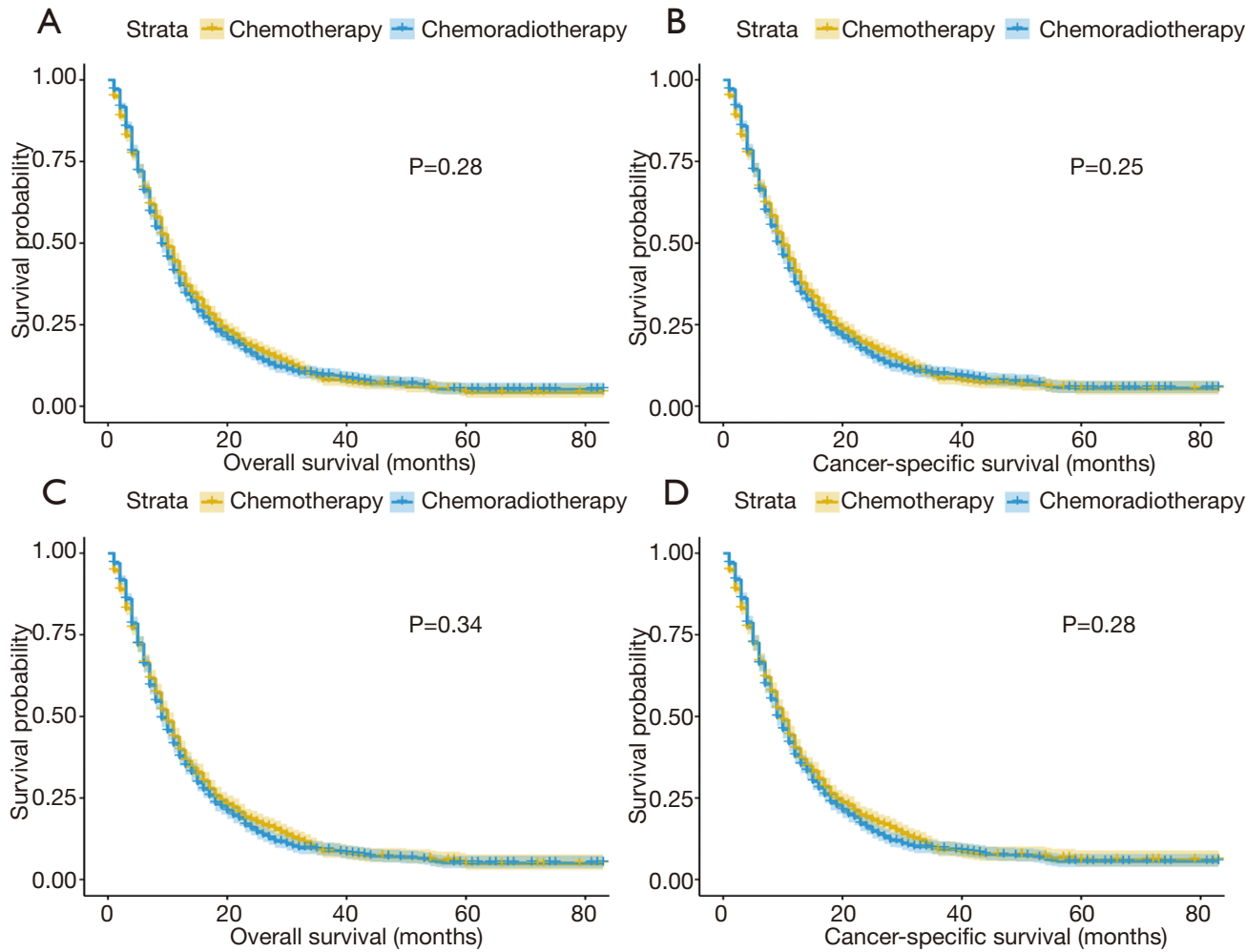

Figure 2 Kaplan-Meier survival analysis before and after propensity score matching (PSM). Overall survival (A) and cancer-specific survival (B) of all patients before PSM. Overall survival (C) and cancer-specific survival (D) of all patients after PSM. PSM, propensity score matching.

underwent CRT had better OS and CSS compared with those receiving CT. In contrast to CRT, perioperative CT improves OS and CSS for EAC patients. In addition, we identified un-partnered, male and ESCC are independent risk factors for $\mathrm{mEC}$.

Although the treatment regimens for $\mathrm{mEC}$ have undergone drastic changes, the standard treatment is yet to be optimized. According to the treatment guidelines in the west, CRT is an acceptable treatment for unresectable mEC $(8,9)$. Tanaka et al. (5) suggested that multimodality therapy, which included CT, radiotherapy and surgery, could improve the outcome in patients with ESCC with distant organ metastasis compared with single-modality. However, mEC is until now not generally treated with a multimodality approach. Guidelines for the National Comprehensive Cancer Network (NCCN) recommend CT as the preferred treatment for mEC patients (9). Notably, our research showed that there were no significant differences in OS and CSS between CT and CRT groups before and after PSM.

In recent years, researchers have found that $\mathrm{EC}$ is a heterogeneous cancer with distinct subtypes $(10,11)$. However, most previous therapies have been developed to treat all subtypes of EC. Specific treatment options based on biology of cancer is important for improving outcomes for patients with advanced EC (12). Related biomarkers may help to further personalize radiotherapy and chemotherapy choices (13). Our analysis revealed that the pathological type is potential marker that can distinguish treatment options, hence can prolong life expectancy. Previous studies revealed that ESCC was more sensitive to radiotherapy $(14,15)$, and patients with ESCC has a higher rate of pathological complete response to CRT (16). Normal cells undergo DNA repair through G1/S phase arrest after radiation, and the key gene in this process is wild-type TP53 $(17,18)$. However, multiple studies have confirmed that the high mutation rate of TP53 in patients with ESCC $(19,20)$. Therefore, ESCC is more sensitive to radiotherapy. Steins et al. (21) reported that CRT may contribute to resistant metastatic disease in EAC patients by inducing epithelial-to-mesenchymal transition. CRT may lead to more radiation-related complications, such as pneumonia, 
Table 2 Multivariate Cox regression analysis for OS and CSS in overall patient cohort

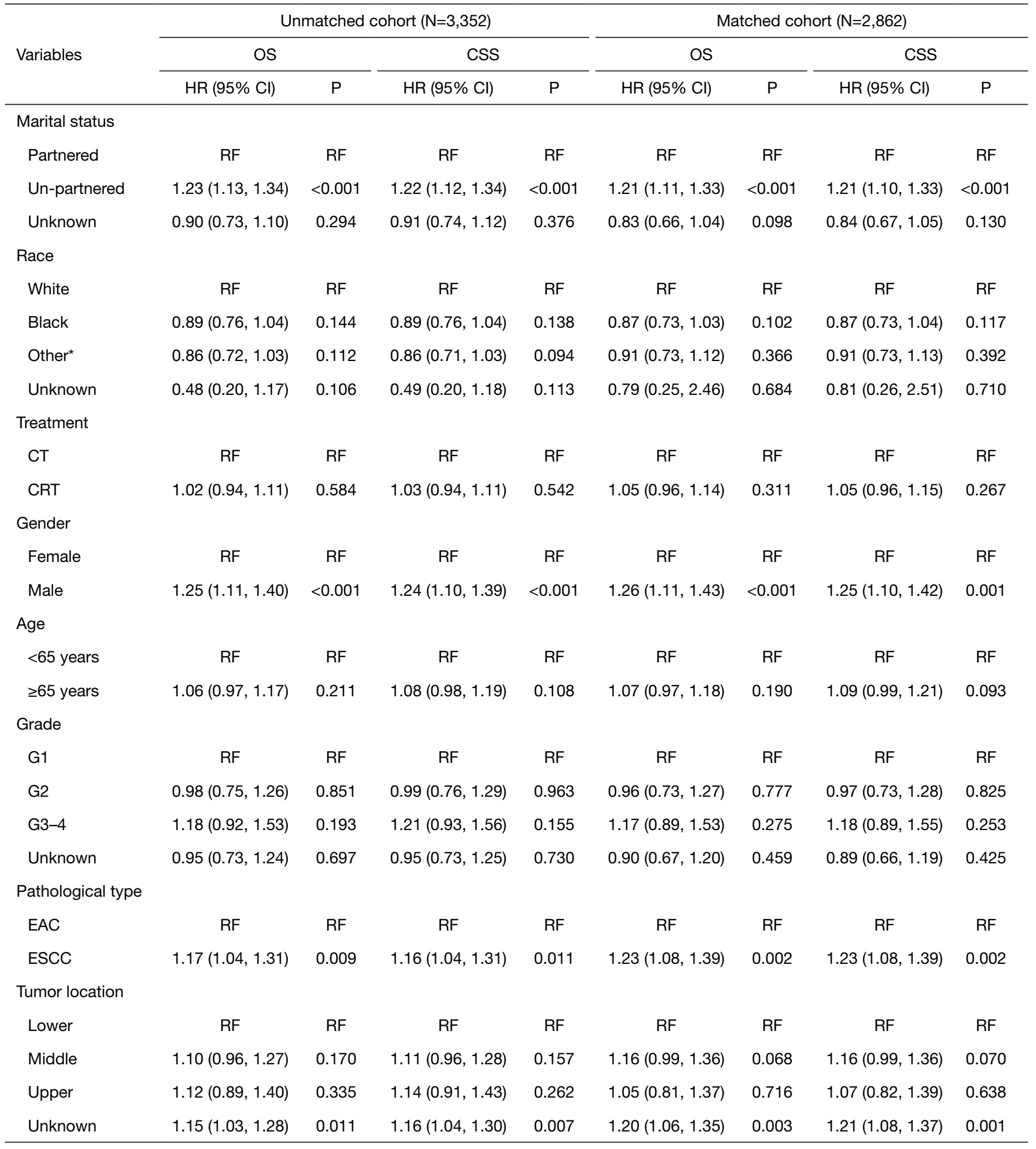

*, Asian, Pacific Islander and American Indian, Alaska Native. RF, reference; OS, overall survival; CSS, cancer-specific survival; HR, hazard ratios; Cl, confidence interval; CT, chemotherapy; CRT, chemoradiotherapy; ESCC, esophageal squamous cell carcinoma; EAC, esophageal adenocarcinoma. 


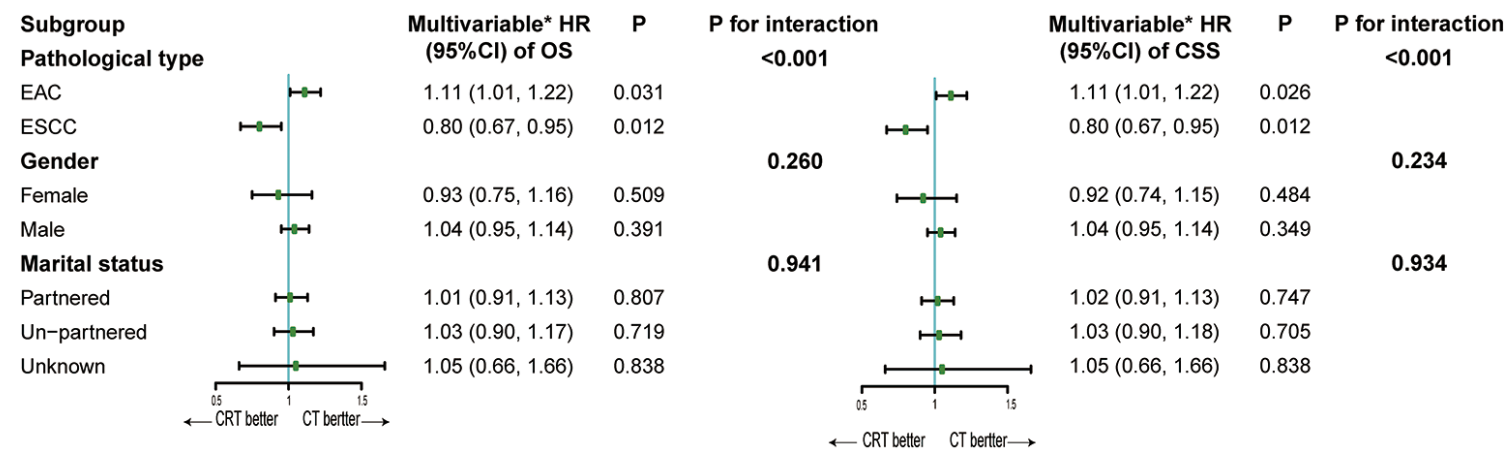

Figure 3 Hazard ratio comparing OS/CSS between chemotherapy group and chemoradiotherapy group according to pathological type, gender and marital status before PSM. *, multivariate analysis adjusted by marital status, race, gender, pathological type, age, grade and tumor location. HR, hazard ratios; CI, confidence interval; CT, chemotherapy; CRT, chemoradiotherapy; ESCC, esophageal squamous cell carcinoma; EAC, esophageal adenocarcinoma; PSM, propensity score matching; OS, overall survival; CSS, cancer-specific survival.
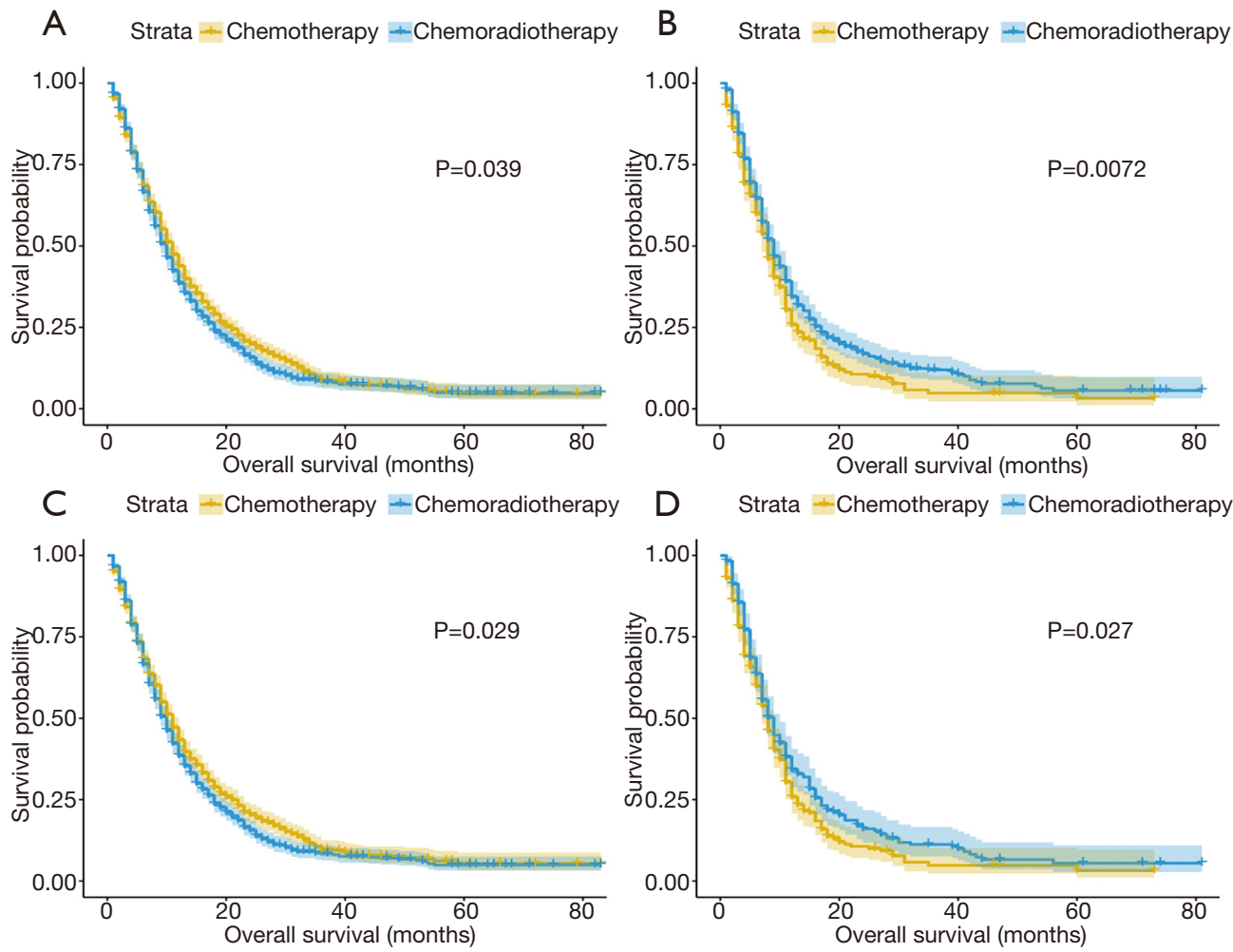

Figure 4 Kaplan-Meier overall survival analysis stratified by pathological type. Overall survival between chemotherapy and chemoradiotherapy groups before PSM in EAC subgroup (A) and ESCC subgroup (B). Overall survival between chemotherapy and chemoradiotherapy groups after PSM in EAC subgroup (C) and ESCC subgroup (D). PSM, propensity score matching; ESCC, esophageal squamous cell carcinoma; EAC, esophageal adenocarcinoma. 

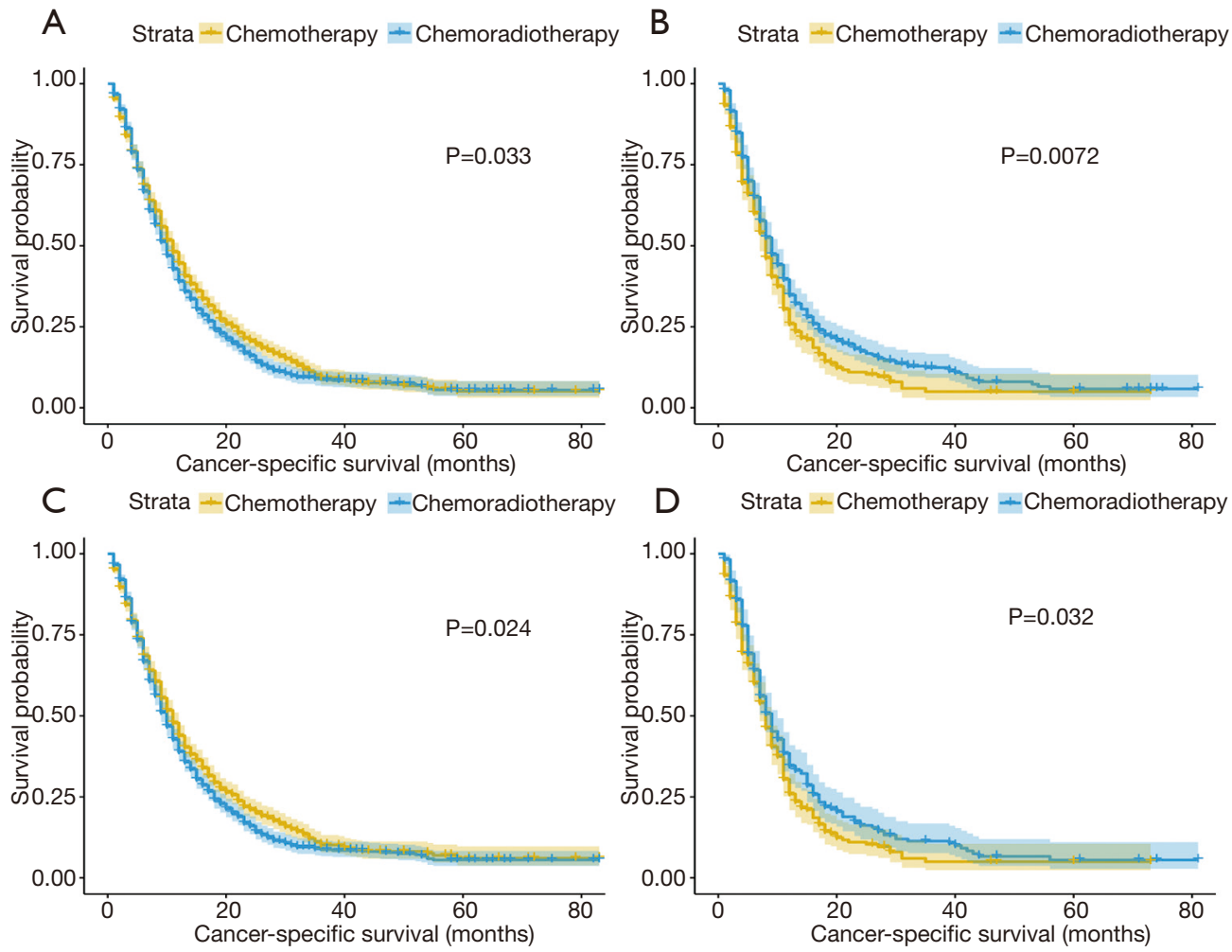

Figure 5 Kaplan-Meier cancer-specific survival analysis stratified by pathological type. Cancer-specific survival comparisons between patients with chemotherapy and chemoradiotherapy before PSM in EAC subgroup (A) and ESCC subgroup (B). Cancer-specific survival comparisons between patients with chemotherapy and chemoradiotherapy after PSM in EAC subgroup (C) and ESCC subgroup (D). PSM, propensity score matching; ESCC, esophageal squamous cell carcinoma; EAC, esophageal adenocarcinoma.

acute respiratory distress syndrome, anastomotic leakage, and cardiac complications (22-24), which could present an additional risk. In addition, previous studies had revealed that CRT had more costs compared with CT alone (25). That may be important reasons why CT alone is more suitable for metastatic EAC.

In line with previous studies (26-30), we also found that marital status, gender and pathological type were associated with prognosis of $\mathrm{mEC}$. Marital status plays a significant role in the prognosis of various cancers $(31,32)$. Patients with severe illness are less likely to get married and have a higher risk of divorce (33). Better health-related support from a partner may contribute to better prognosis.

Our study had some limitations that should be taken into account. The EC data in this paper were all from the publicly accessible SEER database, so we could not control the quality of the original data. Besides, the SEER database lacks information on radiation dose, quality of life, immunotherapy and recurrence data, which may affect our results. As far as we know, this is the first study to explore whether survival differences existed between patients with mEC undergoing CT and CRT. This study is a retrospective, non-randomized analysis that needs further research for verification.

\section{Conclusions}

We find that CT-receiving EAC patients have favorable OS and CSS. However, patients undergoing CT have worse CSS and OS compared with patients who have CRT in the ESCC subgroup. We recommend that as further efforts are made to improve the prognosis of patients with $\mathrm{mEC}$, treatment plans should be tailored to the biological characteristics of an individual's cancer.

\section{Acknowledgments}

We thank Jeremiah Machuki for language editing. 
Funding: This work was supported by the Health Bureau of Chongqing (20121015) and Chongqing Medical University Scholarship Fund for Development of Young Talents (XRJH201905).

\section{Footnote}

Reporting Checklist: The authors have completed the STROBE reporting checklist. Available at http://dx.doi. org/10.21037/apm-20-2126

Peer Review File: Available at http://dx.doi.org/10.21037/ apm-20-2126

Conflicts of Interest: All authors have completed the ICMJE uniform disclosure form (available at http://dx.doi. org/10.21037/apm-20-2126). The authors have no conflicts of interest to declare.

Etbical Statement: The authors are accountable for all aspects of the work in ensuring that questions related to the accuracy or integrity of any part of the work are appropriately investigated and resolved. The study was conducted in accordance with the Declaration of Helsinki (as revised in 2013). Demographic and clinicopathological data extracted from SEER database, and all data included are anonymous. This study has been approved to be exempted research for no human subject involved by the Ethics Committee of the First Affiliated Hospital of Chongqing Medical University.

Open Access Statement: This is an Open Access article distributed in accordance with the Creative Commons Attribution-NonCommercial-NoDerivs 4.0 International License (CC BY-NC-ND 4.0), which permits the noncommercial replication and distribution of the article with the strict proviso that no changes or edits are made and the original work is properly cited (including links to both the formal publication through the relevant DOI and the license). See: https://creativecommons.org/licenses/by-nc-nd/4.0/.

\section{References}

1. Lagergren J, Smyth E, Cunningham D, et al. Oesophageal cancer. Lancet 2017;390:2383-96.

2. Shaheen O, Ghibour A, Alsaid B. Esophageal Cancer Metastases to Unexpected Sites: A Systematic Review. Gastroenterol Res Pract 2017;2017:1657310.
3. Enzinger PC, Mayer RJ. Esophageal cancer. N Engl J Med 2003;349:2241-52.

4. Dubecz A, Gall I, Solymosi N, et al. Temporal trends in long-term survival and cure rates in esophageal cancer: a SEER database analysis. J Thorac Oncol 2012;7:443-7.

5. Tanaka T, Fujita H, Matono S, et al. Outcomes of multimodality therapy for stage IVB esopahageal cancer with distant organ metastasis. Dis Esophagus 2010;23:646-51.

6. Grünberger B, Raderer M, Schmidinger M, et al. Palliative chemotherapy for recurrent and metastatic esophageal cancer. Anticancer research, 2007;27:2705-14.

7. Zhu LL, Yuan L, Wang H, et al. A meta-analysis of concurrent chemoradiotherapy for advanced esophageal cancer. PloS One 2015;10:e128616.

8. Ajani JA, D'Amico TA, Almhanna K, et al. Esophageal and esophagogastric junction cancers, version 1.2015. J Natl Compr Canc Netw 2015;13:194-227.

9. Lordick F, Mariette C, Haustermans K, et al. Oesophageal cancer: ESMO Clinical Practice Guidelines for diagnosis, treatment and follow-up. Ann Oncol 2016;27:v50-7.

10. Yip C, Davnall F, Kozarski R, et al. Assessment of changes in tumor heterogeneity following neoadjuvant chemotherapy in primary esophageal cancer. Dis Esophagus 2015;28:172-9.

11. Yip C, Landau D, Kozarski R, et al. Primary esophageal cancer: heterogeneity as potential prognostic biomarker in patients treated with definitive chemotherapy and radiation therapy. Radiology 2014;270:141-8.

12. Lee S, Cohen DJ. Pharmacotherapy for metastatic esophageal cancer: where do we need to improve? Expert Opin Pharmacother 2019;20:357-66.

13. Courrech Staal EF, Aleman BM, van Velthuysen ML, et al. Chemoradiation for esophageal cancer: institutional experience with three different regimens. Am J Clin Oncol 2011;34:343-9.

14. Zhang R, Jia M, Li P, et al. Radiotherapy improves the survival of patients with metastatic esophageal squamous cell carcinoma: a propensity score matched analysis of Surveillance, Epidemiology, and End Results database. Dis Esophagus 2019. doi:10.1093/dote/doy074.

15. Rackley T, Leong T, Foo M, et al. Definitive chemoradiotherapy for oesophageal cancer - a promising start on an exciting journey. Clin Oncol (R Coll Radiol) 2014;26:533-40.

16. van Hagen $\mathrm{P}$, Hulshof MC, van Lanschot JJ, et al. Preoperative chemoradiotherapy for esophageal or junctional cancer. N Engl J Med 2012;366:2074-84. 
17. Kuroda K, Hibi D, Ishii Y, et al. Role of p53 in the progression from ochratoxin A-induced DNA damage to gene mutations in the kidneys of mice. Toxicol Sci 2015;144:65-76.

18. Pellegata NS, Antoniono RJ, Redpath JL, et al. DNA damage and p53-mediated cell cycle arrest: a reevaluation. Proc Natl Acad Sci U S A 1996;93:15209-14.

19. Egashira A, Morita M, Kakeji Y, et al. p53 gene mutations in esophageal squamous cell carcinoma and their relevance to etiology and pathogenesis: results in Japan and comparisons with other countries. Cancer Sci 2007;98:1152-6.

20. Deng J, Chen H, Zhou D, et al. Comparative genomic analysis of esophageal squamous cell carcinoma between Asian and Caucasian patient populations. Nat Commun 2017;8:1533.

21. Steins A, Ebbing EA, Creemers A, et al. Chemoradiation induces epithelial-to-mesenchymal transition in esophageal adenocarcinoma. Int J Cancer 2019;145:2792-803.

22. Wilke TJ, Bhirud AR, Lin C. A review of the impact of preoperative chemoradiotherapy on outcome and postoperative complications in esophageal cancer patients. Am J Clin Oncol 2015;38:415-21.

23. Luu TD, Gaur P, Force SD, et al. Neoadjuvant chemoradiation versus chemotherapy for patients undergoing esophagectomy for esophageal cancer. Ann Thorac Surg 2008;85:1217-23.

24. Hoeppner J, Zirlik K, Brunner T, et al. Multimodal treatment of locally advanced esophageal adenocarcinoma: which regimen should we choose? Outcome analysis of perioperative chemotherapy versus neoadjuvant chemoradiation in 105 patients. J Surg Oncol 2014;109:287-93.

Cite this article as: Shao Y, Zhang M, Ye L, Chen D, Wu QC, Zhang C. Survival differences between chemotherapy and chemoradiotherapy in metastatic esophageal cancer: a propensity score-matched study based on the SEER database. Ann Palliat Med 2021;10(4):3826-3835. doi: 10.21037/apm-20-2126
25. Kuppusamy M, Sylvester J, Low DE. In an era of health reform: defining cost differences in current esophageal cancer management strategies and assessing the cost of complications. J Thorac Cardiovasc Surg 2011;141:16-21.

26. Tang X, Zhou X, Li Y, et al. A Novel Nomogram and Risk Classification System Predicting the Cancer-Specific Survival of Patients with Initially Diagnosed Metastatic Esophageal Cancer: A SEER-Based Study. Ann Surg Oncol 2019;26:321-8.

27. Deng J, Chu X, Ren Z, et al. Relationship between T stage and survival in distantly metastatic esophageal cancer: A STROBE-compliant study. Medicine (Baltimore) 2020;99:e20064.

28. Liu M, Wang C, Gao L, et al. A nomogram to predict long-time survival for patients with M1 diseases of esophageal cancer. J Cancer 2018;9:3986-90.

29. Du L, Kim JJ, Chen B, et al. Marital status is associated with superior survival in patients with esophageal cancer: a Surveillance, Epidemiology, and End Results study. Oncotarget 2017;8:95965-72.

30. Zhang QW, Lin XL, Zhang CH, et al. The influence of marital status on the survival of patients with esophageal cancer: a population-based, propensity-matched study. Oncotarget 2017;8:62261-73.

31. Aizer AA, Chen MH, McCarthy EP, et al. Marital status and survival in patients with cancer. J Clin Oncol 2013;31:3869-76.

32. Li Q, Gan L, Liang L, Li X, et al. The influence of marital status on stage at diagnosis and survival of patients with colorectal cancer. Oncotarget 2015;6:7339-47.

33. Williams K, Umberson D. Marital status, marital transitions, and health: a gendered life course perspective. J Health Soc Behav 2004;45:81-98. 
A

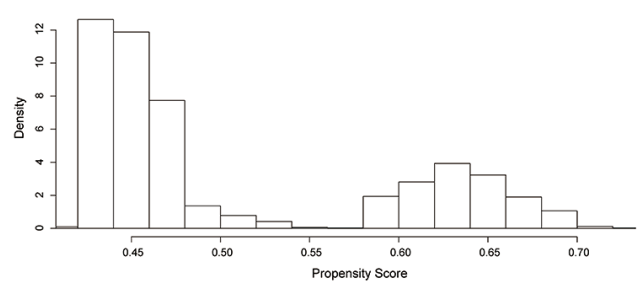

C

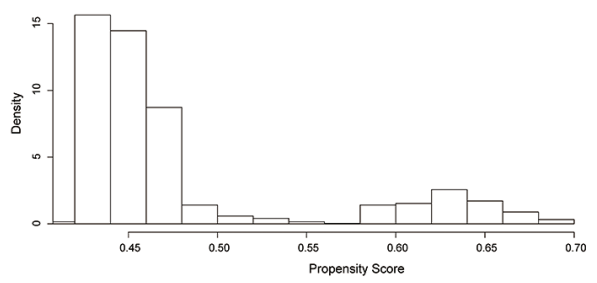

B

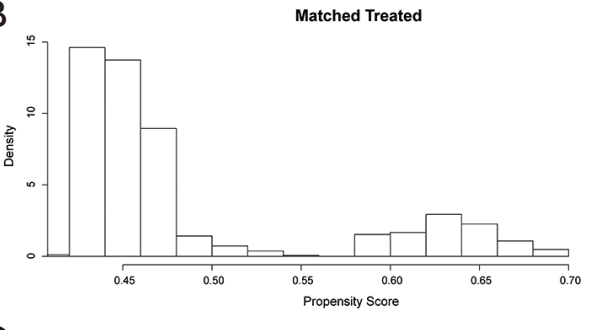

D

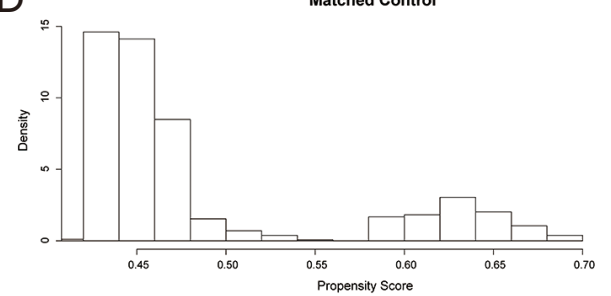

Figure S1 Distribution of propensity score before and after propensity score analysis. (A,C) Distribution of propensity score for patients with chemotherapy (A) and chemoradiotherapy (C) before matching procedure. (B,D) Distribution of propensity score after propensity score matching. 